\title{
Inhibition of the PI3K/AKT Signaling Pathway \\ or Overexpression of Beclin1 Blocks Reinfection of Streptococcus pneumoniae After Infection of Influenza A Virus in Severe Community-Acquired Pneumonia
}

\author{
Zhaohui Yang, ${ }^{1,4}$ Xiaoguang Zou, ${ }^{2}$ Peiqing Feng, ${ }^{3}$ Huaibing Zhan, ${ }^{1}$ Dani Xiong, ${ }^{1}$ and \\ Jianmin Lang ${ }^{1}$
}

\begin{abstract}
Streptococcus pneumoniae (S. pneumoniae) and viruses are considered as primary risks of community-acquired pneumonia (CAP), and the effects of co-infection bacterial and virus in the prognosis of patients with severe CAP (SCAP) are poorly described. Therefore, this study is conducted to investigate the regulation of Beclin1$\mathrm{PI} 3 \mathrm{~K} / \mathrm{AKT}$ axis in reinfection of $S$. pneumoniae after influenza A virus in mice model of bronchoalveolar lavage fluid (BALF). Samples of sputum and BALF were collected from patients with SCAP for etiological detection. The expression of each gene was determined by RT-qPCR and western blot analysis. Influenza A/PR/8/34 and $S$. pneumoniae were used to establish the mice model of reinfection pneumonia. The virus quantity, expression levels of inflammatory factors, bacterial load, and myeloperoxidase (MPO) activity were tested. HE staining was applied to observe histopathology of lung tissue. The expression of Beclin1 was downregulated and the $\mathrm{PI} 3 \mathrm{~K} / \mathrm{AKT}$ pathway was activated in viral pneumonia. In vivo experiment, the reinfection of $S$. pneumoniae following influenza A virus infection increased the number of $S$. pneumoniae population, the activity of MPO, and the expression of TNF- $\alpha$, IL-6, and IFN- $\gamma$ in BALF of mice. In contrast, inhibition of the PI3K/AKT pathway or overexpression of Beclin1 reduced the number of $S$. pneumoniae population, the activity of MPO, and the expression of TNF- $\alpha$, IL- 6 , and IFN- $\gamma$ in BALF of mice reinfected with S. pneumoniae after influenza A virus infection. Collectively, our study demonstrates that inhibition of the PI3K/AKT signaling pathway or overexpressed Beclin1 alleviates reinfection of $S$. pneumoniae after influenza A virus infection in SCAP.
\end{abstract}

\footnotetext{
${ }^{1}$ Department of Respiratory Medicine, Qingdao Huangdao District Central Hospital, No. 9, Huangpujiang Road, Huangdao District, Qingdao, 266555, Shandong Province, People's Republic of China

${ }^{2}$ Intensive Care Unit, Qingdao Huangdao District Central Hospital, Qingdao, 266555, People's Republic of China

${ }^{3}$ Clinical Laboratory, Qingdao Huangdao District Central Hospital, Qingdao, 266555, People's Republic of China
}

\footnotetext{
${ }^{4}$ To whom correspondence should be addressed at Department of Respiratory Medicine, Qingdao Huangdao District Central Hospital, No. 9, Huangpujiang Road, Huangdao District, Qingdao, 266555, Shandong Province, People's Republic of China. E-mail: yangzhaohui_yzh@126.com
} 
KEY WORDS: severe community-acquired pneumonia; PI3K/AKT signaling pathway; Beclin1; Streptococcus pneumoniae; influenza A virus infection.

\section{INTRODUCTION}

Community-acquired pneumonia (CAP) is a serious infectious disease accompanied with high morbidity and mortality, and has a mortality of $30-50 \%[1,2]$. It is defined as a common infection which is potentially life threatening, particularly in older adults along with those with comorbid disease [3]. Severe CAP (SCAP) has generally been defined as CAP requiring admission to the intensive care unit due to invasive mechanical ventilation or septic shock requiring vasopressors [4-6]. It is related to severe mortality and approximately $16-36 \%$ patients with SCAP may die in a short time [7]. It is reported that about one-third of patients with CAP requiring hospitalization present with co-infection of both bacteria and virus, and most of them are Streptococcus pneumoniae (S. pneumoniae) and influenza [8]. The main bacterial causes of CAP are well described, with $S$. pneumoniae acting as the most significant pathogen in all age groups [9]. Viruses, such as influenza A and B viruses, parainfluenza viruses as well as adenoviruses, are also considered as important causes of CAP [10]. Currently, proper antibiotics, simple analgesics accompanied with adequate rest are the major medication against pneumonia [11]. And the macrolide antibiotics have been demonstrated to be widely applied to treat CAP (including pneumococcal pneumonia) due to their excellent inhibition against S. pneumoniae and atypical organisms [12]. Nevertheless, the prevalence of influenza A (H1N1) virus-associated pneumonia and the role of bacterial co-infection in the prognosis of patients are poorly described in the general population [13].

Flexible bronchoscopy combined with bronchoalveolar lavage (BAL) is a viable alternative for sputum acquisition if noninvasive samples are unobtainable from the lower respiratory tract [14]. Pathogens detected in BAL can be verified by easy molecule biological methods, which can be used to improve the sensitivity and accuracy of diagnostic studies in patients with CAP who are nonresponsive to empiric antibiotic therapy [15]. However, the molecular mechanism of pathology by these pathogens remains unclear. Pneumonia is per se an inflammatory condition caused by multiple factors. It has been reported that pneumonia is strongly associated with inflammation caused by autophagy and apoptosis [16]. Beclin1, a mammalian homolog of the yeast Atg6/Vacuolar protein sorting
30 (Vps30) protein, is suggested to play a vital role in macroautophagy and vacuolar protein sorting [17]. Meanwhile, Beclin 1 is necessary for the development of normal mammalian and it has been demonstrated to play a pivotal role in pathogenesis, including cancer [18]. The phosphorylation of Beclin1 mediated by protein kinase B (AKT) functions in oncogenesis and autophagy inhibition [19]. Meanwhile, the phosphoinositide 3-kinase (PI3K)/AKT signaling pathway mediated by a pathogen contributes to coordination of actin rearrangement, thereby leading to internalization of the organism [20]. A study has suggested that the activated PI3K/AKT/mammalian target of rapamycin (mTOR) signaling pathway is implicated in lung tumorigenesis and it has been related to high-grade tumors and advanced diseases [21]. In conclusion, this study is carried out to discuss the role of Beclin1-PI3K/ AKT axis in reinfection of S. pneumoniae after influenza A virus.

\section{MATERIALS AND METHODS}

\section{Study Subjects}

From April 2012 to December 2015, 60 patients with CAP (37 males and 23 females who aged 1845 years with an average age of 34 years) in Qingdao Huangdao District Central Hospital were collected into our study. The included patients met the criteria for CAP and the criteria for SCAP in Infectious Diseases Society of America/American Thoracic Society guidelines in 2007 [6]. The patients were excluded from our study who met the following criteria: patients with lung neoplasms, noninfectious pulmonary interstitial diseases, pulmonary edema, atelectasis, pulmonary embolism, pulmonary eosinophil infiltration, and pulmonary vasculitis; patients with acquired immune deficiency syndrome or human immunodeficiency virus infection; patients were diagnosed with tuberculosis or nontuberculosis mycobacterium infection; patients admitted to a nursing home within three months or who have used antibiotics within three months to consider cases of healthcareassociated pneumonia; patients showed lower respiratory tract infection $48 \mathrm{~h}$ after admission to consider cases of nosocomial pneumonia. An electro-fiberoptic 
bronchoscopy was used to lavage the lung segment and sublung segment of the selected patients with saline water, and $20 \mathrm{~mL}$ bronchoalveolar lavage fluid (BALF) and sputum samples from patients with CAP were collected for further detection. Additionally, BALF samples, obtained from foreign bodies in the bronchus, from 15 healthy controls were collected as controls, including nine males and six females, whose age was between 18 and 45 years, with the mean age of 33 years.

\section{Etiological Detection}

Microbes from sputum samples and BALF solution were cultured. The nested polymerase chain reaction (PCR) was conducted to detect $S$. pneumoniae [22]. A preliminary in vitro study verified that the lower limit of detection was $\leq 10$ colony-forming units/mL [23], but the specificity with no identifiable respiratory disorders was $100 \%$ among control subjects. A study has shown that the same primer has $100 \%$ specificity when detecting many pathogens in vitro [22]. The genomic DNA of BAA-255D5 S. pneumoniae (from American Type Culture Collection [ATCC]) was used as a positive control for PCR. Respiratory viruses were determined by a multiplex reverse transcription PCR assay using a Seeplex RV15 ACE detection kit (Seegene Inc., Seoul, Korea) and/or shell vial culture. Strain ATCC $33533 H$. influenzae was set as a positive control.

\section{Reverse Transcription Quantitative PCR}

Total RNA was extracted using Trizol (Invitrogen, Carlsbad, CA, USA). The integrity and purity of RNA were determined by agarose gel electrophoresis. Primescript $^{\mathrm{TM}}$ RT reagent RT kit (RRO37A, TaKaRa, Dalian, Liaoning, China) was used for RT. The target gene and internal reference gene (glyceraldehyde phosphate dehydrogenase [GAPDH]) were amplified by fluorescence qPCR (Applied Biosystems, Carlsbad, CA, USA). The reaction system of PCR was $25 \mu \mathrm{L}$. The mRNA expression of gene was calculated by $2^{-\Delta \Delta \mathrm{Ct}}$ method [24]. The primer sequence was shown in Table 1.

\section{Western Blot Analysis}

The cells were lysed with radioimmunoprecipitation assay (RIPA) lysate containing protease inhibitor. Protein concentration was determined by bicinchoninic acid (BCA) assay. The protein samples $(20 \mu \mathrm{g})$ bore sodium dodecyl sulfate polyacrylamide gel electrophoresis, and
Table 1. Primer Sequence for RT-qPCR

\begin{tabular}{ll}
\hline Gene & Primer sequence \\
\hline Beclin1 (human) & F: 5'-TGTCACCATCCAGGAACTCA-3' \\
& R: 5'-CTGTTGGCACTTTCT-GTGGA-3' \\
Beclin1 (mouse) & F: 5'-ATGGAGGGGTCTAAGGCGTC-3' \\
& R: 5'-TGGGCTGTGGTAAGTAATGGA-3' \\
GAPDH & F: 5'-GCACCGTCAAGGCTGAGAAC-3' \\
& R: 5'-ATGGTGGTGAAGACGCCAGT-3' \\
\hline
\end{tabular}

Note: $F$, forward; $R$, reverse; $R T-q P C R$, reverse transcription quantitative polymerase chain reaction; GAPDH, glyceraldehyde phosphate dehydrogenase

then were electro-transferred to the polyvinylidene fluoride (PVDF) membrane. The PVDF membrane was sealed with $5 \%$ skim milk powder for two $\mathrm{h}$ and then incubated with the primary antibody at $4{ }^{\circ} \mathrm{C}$ overnight. The primary antibodies included PI3K (ab151549, 1:1000), p-PI3K (ab182651, 1:1000), AKT (ab8805, 1:500), p-AKT (ab38449, 1:1000), mTOR (ab2732, 1:2000), and p-mTOR (ab137133, 1:1000). All these antibodies were purchased from Abcam Inc. (Cambridge, MA, USA). After washing with $0.1 \%$ Trisbuffered saline and Tween-20 (TBST) three times, the membrane was incubated in horseradish peroxidase (HRP)-labeled goat anti rabbit antibody to immunoglobulin G (IgG) (Abcam Inc., Cambridge, MA, USA) at $37^{\circ} \mathrm{C}$ for one h. Finally, the membrane was covered by diaminobenzidine (DAB) solution and exposed by enhanced chemiluminescence (ECL).

\section{Establishment of an Animal Model}

A total of 120 healthy specific pathogen-free male C57BL/6J mice of eight to ten weeks and weighing 20-30 g were purchased from Better Bioengineering Co., Ltd., (Nanjing, Jiangsu, China). Influenza A/PR/8/34 (VR-95; ATCC, Rockville, MD) was allowed to grow in LLC-MK2 cells (RIVM, Bilthoven, Netherlands). The virus was harvested by repeated freezing and thawing, and then centrifuged at $680 \times g$ for $10 \mathrm{~min}$. The $50 \%$ tissue culture infective dose $\left(\mathrm{TCID}_{50}\right)$ in virus solution was calculated by titration in LLC-MK2 cells [25]. The uninfected cell cultures were utilized for preparation of control inoculants. The stored cells were not contaminated by other respiratory viruses including the influenza $\mathrm{B}$ virus, human parainfluenza 1, 2, 3, 4A, and 4B, sendai virus, respiratory syncytial virus $A$ and $B$, 
rhinovirus, enterovirus, coronavirus, and adenovirus. Exclusion contamination was determined by PCR or cell culture. The virus and control solution were diluted in phosphate buffer saline (PBS, $\mathrm{pH}=7.4$ ). The primary influenza infection and secondary pneumococcal pneumonia were induced according to previously described methods $[26,27]$.

\section{Animal Treatment}

Mice were allocated into 12 groups $(n=10)$ : control group (without any treatment), Flu group (infected with H1N1), SP group (inoculated with S. pneumoniae), Flu + SP group (inoculated with $S$. pneumoniae after H1N1 infection), dimethyl sulfoxide (DMSO) + Flu + SP group (inoculated with $S$. pneumoniae after H1N1 infection in the process of DMSO treatment), DMSO + SP group (inoculated with $S$. pneumoniae in the process of DMSO treatment), PI3K inhibitor + SP group (inoculated with S. pneumoniae in the process of LY294002 inhibitor treatment), PI3K inhibitor + Flu + SP group (inoculated with $S$. pneumoniae after H1N1 infection in the process of LY294002 inhibitor treatment), oenegative control $(\mathrm{NC})+\mathrm{SP}$ group (inoculated with $S$. pneumoniae in the process of oe-NC lentivirus treatment), oe-Beclin 1 + SP group (inoculated with $S$. pneumoniae in the process of oe-Beclin1 lentivirus treatment), oe-NC + Flu + SP group (inoculated with $S$. pneumoniae after $\mathrm{H} 1 \mathrm{~N} 1$ infection in the process of oe-NC lentivirus treatment), and oe-Beclin $1+$ Flu + SP group (inoculated with $S$. pneumoniae after H1N1 infection in the process of oe-Beclin1 lentivirus treatment). Both oe-NC and oe-Beclin1 lentivirus vectors were constructed by Shanghai GenePharma Co., Ltd., (Shanghai, China) using LV5-GFP. The lentivirus was packaged with $293 \mathrm{~T}$ cells, and $293 \mathrm{~T}$ cells were cultured on the RPMI-1640 medium containing $10 \%$ fetal bovine serum (FBS) and were subcultured every other day. From the first two weeks after inoculation of H1N1 virus or $S$. pneumoniae, the lentivirus vectors $(5 \times$ $\left.10^{8} \mathrm{pfu} / 100 \mu \mathrm{L}\right)$ or LY294002 $(40 \mu \mathrm{M})$ were introduced into the lungs of mice by nasal inhalation two times a week for a total of four weeks.

\section{BALF Solution Collection}

Trachea was exposed through midline incision and intubated with aseptic 22 Abbocath-T catheter (Abbott, Sligo, Ireland). BAL was performed by injecting two parts of $0.5 \mathrm{~mL}$ aseptic saline into the right lung. The recovered BALF $(0.8 \mathrm{~mL})$ was rotated at $4{ }^{\circ} \mathrm{C}$ for $10 \mathrm{~min}$ at $260 \times \mathrm{g}$, and the precipitate was re-suspended in $0.5 \mathrm{~mL}$ sterile PBS. The total number of cells was counted by a Z2 Particle Counterand Size Analyzer (Beckman-Coulter, Miami, FL, USA). Differential cell counting (Diff-Quick, Baxter, UK) was performed on cell centrifuge smears stained with modified Giemsa stain.

\section{Determination of Virus Quantity}

According to the reported method [28], at the 4th, 8th, 12th, and 14th days after virus infection and $48 \mathrm{~h}$ after pneumococcal infection (i.e., 16th days after virus infection), the viral load was determined by reverse transcription quantitative PCR (RT-qPCR). In short, $5 \mu \mathrm{L}$ of $25 \mu \mathrm{L}$ cDNA template derived from cells in BALF was used for real-time qPCR (ABI PRISM 7700 sequence detection system). The viral load in each sample was calculated using the standard curve of particle counting of influenza viruses (viral particles were counted by an electron microscopy). The primer used was 5-GGACTGCAGCTGAG ACGCT-3 (forward); 5-CATCCTGTTGTATATGAGGC CCAT-3 (reverse); and 5-CTCAGTTATTCTGC TGGTGCACTTGCC-3 (5-FAM labeled probe).

\section{Measure of Bacterial Load}

After 24 or $48 \mathrm{~h}$ of $S$. pneumoniae infection, mice were anesthetized with 3\% pentobarbital sodium (P3761, Sigma-Aldrich, St Louis, MO, USA), and the BALF of mice in each group was obtained. The recovered BALF $(0.8 \mathrm{~mL})$ was rotated at $4{ }^{\circ} \mathrm{C}$ for $10 \mathrm{~min}$ at $260 \times g$, and the precipitate was re-suspended in $0.5 \mathrm{~mL}$ sterile PBS. Subsequently, the suspension was diluted by 10 times, and the final diluent $(50 \mathrm{~mL})$ was coated on a sheep blood agar plate and fixed in $5 \% \mathrm{CO}_{2}$ at $37^{\circ} \mathrm{C}$. Colony-forming units were counted $16 \mathrm{~h}$ later.

\section{Enzyme-Linked Immunosorbent Assay}

Tumor necrosis factor (TNF)- $\alpha$, interleukin (IL)-6, and interferon (IFN)- $\gamma$ enzyme-linked immunosorbent assay (ELISA) kits were purchased from R\&D Systems (Minneapolis, NM, USA) for expression determination of TNF- $\alpha$, IL- 6 , and IFN- $\gamma$ in BALF of each group according to the instructions of the kits.

\section{Determination of Myeloperoxidase Activity}

Determination of the myeloperoxidase (MPO) activity was performed as described earlier [29]. BAL was 
diluted by five times in potassium phosphate buffer $(\mathrm{pH}=$ 6.0) supplemented with $0.5 \%$ cetyltrimethylammonium bromide and $10 \mathrm{mM}$ ethylene diamine tetraacetic acid. The activity of MPO was measured by $\mathrm{H}_{2} \mathrm{O}_{2}$-dependent oxidation of 3,3,5,5-tetramethyl benzidine at $37^{\circ} \mathrm{C}$. The reaction was terminated by adding acetic acid $(0.2 \mathrm{M})$. The amount of transformed 3-3-5-5-tetramethyl benzidine was determined by measuring optical density (OD) value at $655 \mathrm{~nm}$. MPO activity was expressed as units of BAL liquids $/ \mathrm{mL}$. A unit was defined as the amount of MPO required to produce one $\mathrm{OD}_{655}$ unit per minute. The measurement of MPO activity in the BAL solution indicated the activated state of the polymorphonuclear cells presenting in the lung.

\section{Hematoxylin and Eosin Staining}

The lung tissues were fixed with formaldehyde, hydrated with gradient alcohol, cleared in xylene, and sliced to four $\mu \mathrm{m}$. Subsequently, the tissues were dewaxed with xylene, hydrated with gradient alcohol, stained with hematoxylin for $10 \mathrm{~min}$, differentiated with $1 \%$ alcohol hydrochloric acid for $20 \mathrm{~s}$, treated with $1 \%$ ammonia for $30 \mathrm{~s}$, and stained with eosin for three min. Afterwards, the tissues were hydrated with gradient alcohol, cleared in xylene, sealed by neutral balsam, and observed under a microscopy. Lastly, the images were obtained and analyzed under a 40 times ordinary optical microscope (Olympus, Tokyo, Japan).

\section{Statistical Analysis}

Analysis of the data from this study was performed using SPSS 21.0 statistical software (IBM Corp., Armonk, NY, USA). The measurement data were expressed as mean \pm standard deviation. The date with the normal distribution was analyzed by Student's $t$ test between two groups, and the one-way analysis of variance (ANOVA) or repeated measures ANOVA was used in the comparison among multiple groups, followed by the Dunnett post hoc test.
Enumeration data were assessed using chi-square test to evaluate differences among groups. The survival curve was drawn by Kaplan-Meier and the difference of survival rate was scaled by the log-rank test. $p<0.05$ showed significant difference.

\section{RESULTS}

\section{Downregulation of Beclin1 and Activation of the PI3K/ AKT Pathway Are Found in Reinfection Pneumonia}

Bacterial pathogens in BALF collected from patients with CAP were detected, and the results showed that compared with the sputum sample, the positive rate of disease diagnosis was significantly higher by using BALF (Table 2). By detecting the respiratory viruses in the clinical samples of BALF, we found that 31 cases $(51.67 \%)$ were positive in the sputum sample while 45 cases $(75.00 \%)$ were positive in BALF sample $(p<0.05$; Table 3$)$. These results suggested that BALF sampling was superior to traditional sputum sampling in the detection of bacterial pathogens in pneumonia. In order to investigate whether the pathogenesis of reinfection pneumonia was related to the Beclin1-PI3K/AKT signaling pathway, we examined the expression of related genes in alveolar epithelial cells of BALF in 16 viral positive patients. The expression of Beclin1 in BALF was significantly lower than that in sputum of patients with reinfection pneumonia $(p<0.05)$, and the expression of Beclin1 in BALF of viral positive patients further decreased $(p<0.05$; Fig. 1a). Five cases were randomly selected from each group, and then determination of the Beclin1-PI3K/AKT pathway-related proteins (Fig. 1b, c) suggested that in contrast to the normal group, levels of p-PI3K/PI3K, p-AKT/AKT, and p-mTOR/mTOR increased while the protein expression of Beclin1 decreased in the Pne and the Flu + Pne group (all $p<0.05$ ). Relative to the Pne group, levels of p-PI3K/PI3K, p-AKT/AKT, and p$\mathrm{mTOR} / \mathrm{mTOR}$ further increased while the expression of

Table 2. Detection Rate of Streptococcus pneumoniae Between Samples of Sputum and Bronchoalveolar Lavage Fluid in Mice $(n=60)$

\begin{tabular}{lll} 
Causative agent & $\begin{array}{l}\text { Sputum sample } \\
\text { positive (strain) }\end{array}$ & $\begin{array}{l}\text { Bronchoalveolar lavage } \\
\text { fluid positive (strain) }\end{array}$ \\
\hline $\begin{array}{l}\text { Streptococcus pneumoniae } \\
\text { Rate }\end{array}$ & 39 & 53 \\
\hline
\end{tabular}

Note: $n=60$, enumeration data were assessed using chi-square test to evaluate differences among groups 
Table 3. Virus Detection Rate Between Samples of Sputum and Bronchoalveolar Lavage Fluid in Mice $(n=60)$

\begin{tabular}{llll}
\hline Group & $\begin{array}{l}\text { Sputum sample } \\
\text { positive (strain) }\end{array}$ & $\begin{array}{l}\text { Bronchoalveolar lavage } \\
\text { fluid positive (strain) }\end{array}$ & $X^{2}$ \\
\hline Virus & 31 & 45 & 6.065 \\
Rate & $51.67 \%$ & $75.00 \%$ & 0.014 \\
\hline
\end{tabular}

Note: $n=60$, enumeration data were assessed using chi-square test to evaluate differences among groups

Beclin1 further decreased in the Flu + Pne group (all $p<0.05$ ), suggesting that Beclin1 is downregulated and the PI3K/AKT pathway is activated in reinfection pneumonia.

\section{A Mouse Model of Influenza Virus/S. pneumoniae Infection Is Successfully Established}

In order to verify whether the Beclin1-PI3K/AKT signaling pathway was involved in influenza virus/ $S$. pneumoniae reinfection in mice, relevant mouse model was established. The results in the detection of survival rate of mice in each group suggested that during infection, no mice died in the control group or in the Flu group. Compared with the control group and Flu group, the survival rate of mice significantly decreased in the SP group, and further decreased in the Flu + SP group $(p<0.05$; Fig. $2 \mathrm{a})$. The amount of virus detected in the lungs of the mice showed that the number of H1N1 influenza viruses in the Flu and Flu + SP groups increased significantly on the 5th day compared with the 1st day (Fig. 2b). Based on the findings in the detection of the population of $S$. pneumoniae, compared with the 1 st day, the number of $S$. pneumoniae in the SP and Flu + SP groups increased significantly on the 5 th day $(p<0.05)$. And the detective population of $S$. pneumoniae in the Flu + SP group was significantly higher than that in the SP group $(p<0.05$; Fig. $2 c)$. In terms of MPO activity, the measurement results showed that the activity of MPO in the SP and Flu + SP group was significantly higher than that in the control group ( $p<0.05$; Fig. $2 d)$. According to the results observed by hematoxylin and eosin (HE) staining, we found that in the control group, the lung tissue of mice showed normal state. However, in the SP group, inflammatory cell infiltration, alveolar collapse, perivascular, and peribronchiole edema were found in the lung tissue of mice. What is more, the lung injury in the Flu + SP group was more serious than that in the SP group (Fig. 2e). These results suggest that we successfully established a mouse model of influenza virus/S. pneumoniae infection.
A

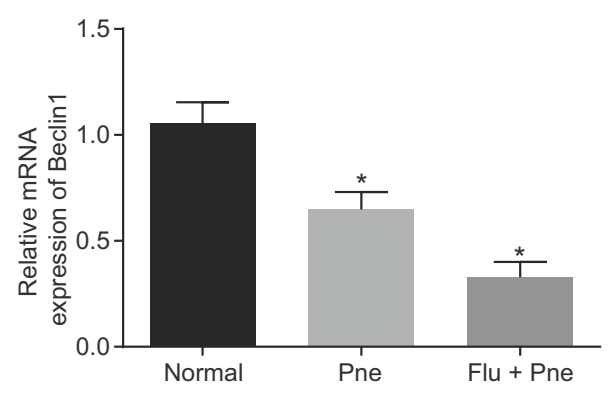

B

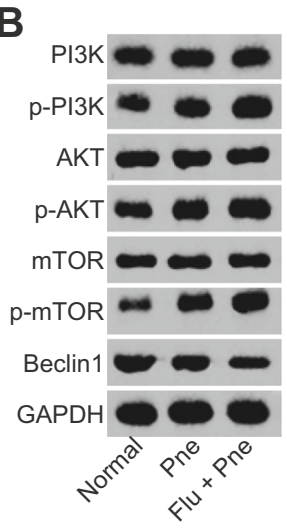

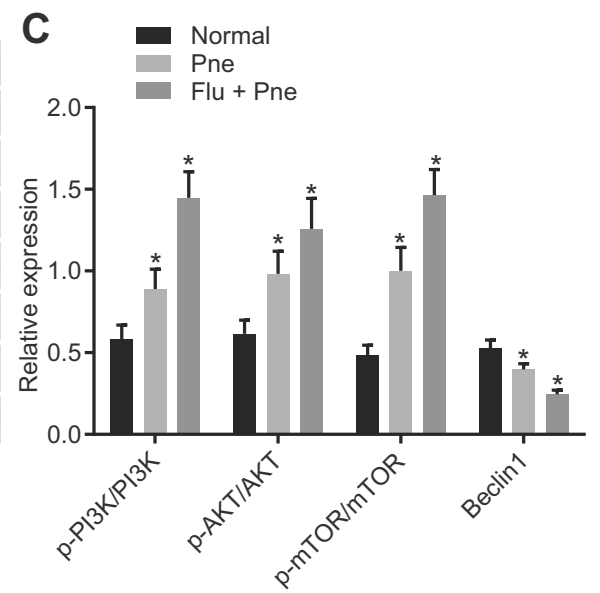

Fig. 1. Downregulation of Beclin1 and activation of the PI3K/AKT signaling pathway in reinfection pneumonia. a Determination of Beclin1 expression in all included samples by RT-qPCR, ${ }^{*} p<0.05 v s$. the normal group. b Determination of PI3K/AKT signaling pathway-related proteins in 15 samples by western blot analysis, ${ }^{*} p<0.05$ vs. the normal group. The measurement data were expressed in the form of mean \pm standard deviation, and the comparison among multiple groups should be based on one-way analysis of variance, followed by Dunnett's post hoc test. The experiment was repeated three times. Normal: $n=15$, Pne: $n=15$, Flu + Pne: $n=45$. RT-qPCR, reverse transcription quantitative polymerase chain reaction. 
A

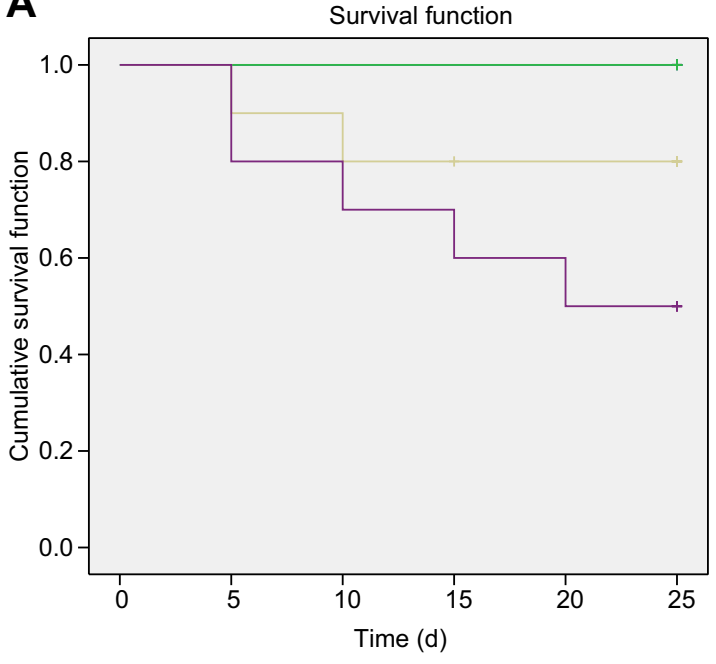

C

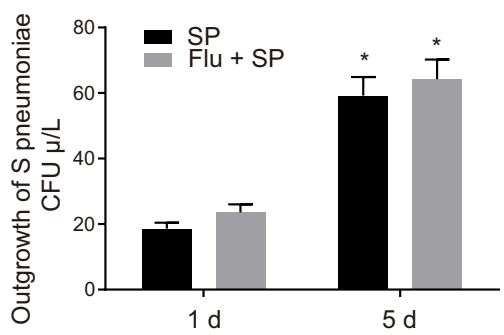

D

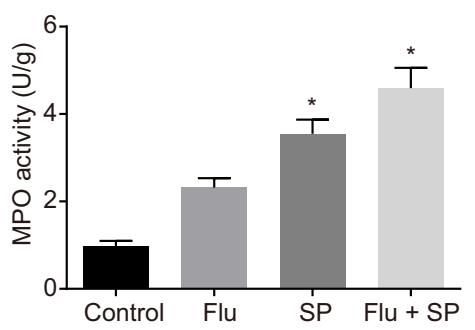

B

Group

$\neg$ Control

$\neg \mathrm{Flu}$

$\rightarrow$ Flu + SP

+ Control-censored

+ Flu-censored

+ Flu + SP-censored



E Control Flu
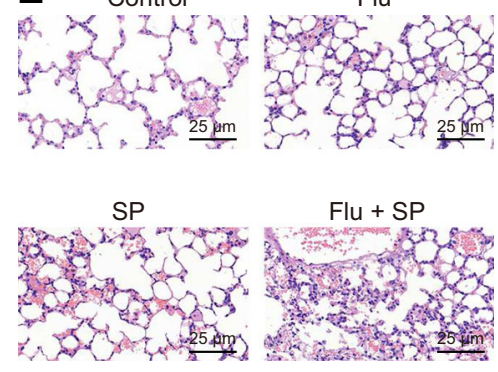

Fig. 2. Successful establishment of mouse model of influenza virus/S. pneumoniae infection. a Survival analysis of mice in each group, ${ }^{*} p<0.05 v s$. the Flu + SP group. b The detection of the amount of virus in the lungs of the mice, ${ }^{*} p<0.05$ vs. the 1 st day. c Detection of Streptococcus pneumoniae in bronchoalveolar lavage fluid of mice, ${ }^{*} p<0.05 v s$. the 1 st day. $\mathbf{d}$ Measurement of MPO activity in bronchoalveolar lavage fluid of mice, ${ }^{*} p<0.05 v s$. the control group. e Observation of pathological changes of lung tissue in mice by HE staining $(\times 400)$. The measurement data were expressed in the form of mean \pm standard deviation, and the comparison among multiple groups should be based on one-way analysis of variance, followed by Dunnett's post hoc test. The experiment was repeated 3 times. $N=10$. SP, Streptococcus pneumoniae; MPO, myeloperoxidase; HE, hematoxylin and eosin.

\section{Activated PI3K/AKT Signaling Pathway and Aggra- vated Cell Inflammation Are Presented in Reinfection of S. pneumoniae After Influenza Virus Infection in a Mouse Model}

To investigate whether the Beclin1-PI3K/AKT signaling pathway was involved in the disease process, we examined the expression of related genes. The results based on the western blot analysis indicated that relative to the control group, levels of p-PI3K/PI3K, p-AKT/AKT, and $\mathrm{p}-\mathrm{mTOR} / \mathrm{mTOR}$ significantly increased while Beclin1 expression decreased significantly in the Flu, SP, and Flu + SP groups (all $p<0.05$ ). Compared with the Flu and SP groups, levels of p-PI3K/PI3K, p-AKT/AKT, and p$\mathrm{mTOR} / \mathrm{mTOR}$ in the Flu + SP group further increased, and the expression of Beclin1 protein further decreased (all $p<0$. 05; Fig. 3a, b). The expression of Beclin1 in lung tissue of each group of mice was examined by immunohistochemistry, and the results were consistent with those of western blot analysis (Fig. 3c, d). These results suggest that the Beclin1-PI3K/AKT signaling pathway is involved in the process of influenza A virus infection. The expression of inflammatory factors in BALF of each group was detected. The findings revealed that in contrast to the control group, the expression of TNF- $\alpha$, IL-6, and IFN$\gamma$ in the SP and Flu + SP group significantly increased (all $p<0.05$ ). Meanwhile, the expression of the above inflammatory factors in the Flu + SP group was significantly higher than those in the SP group (all $p<0.05$; Fig. 3e-g). Altogether, it is suggested that PI3K/AKT signaling pathway is activated and cell inflammation is aggravated in reinfection of $S$. pneumoniae after influenza virus infection in a mouse model. 


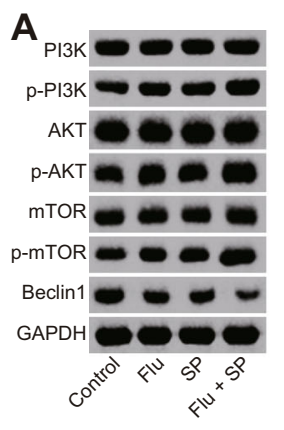

B

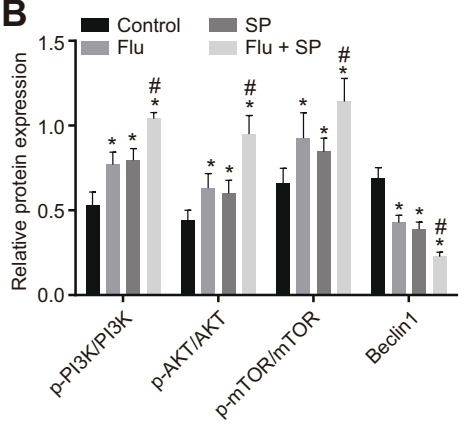

C
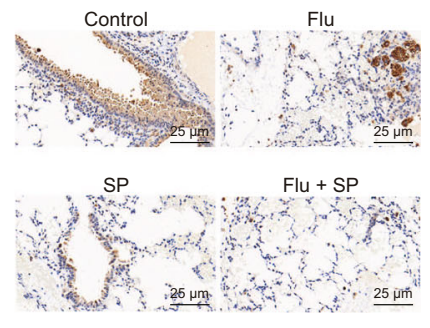

D

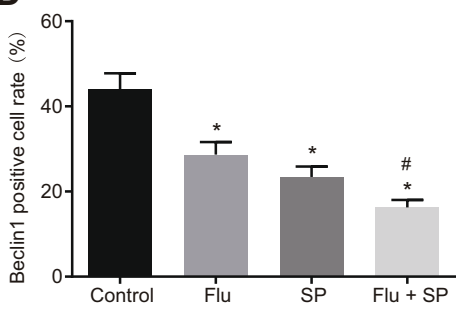

$E$

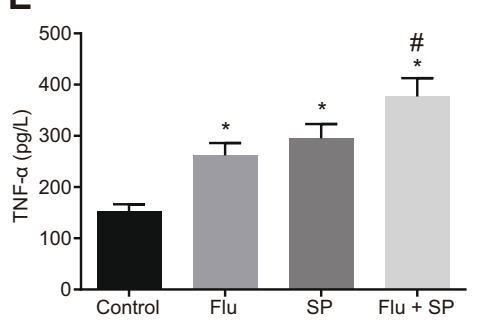

$\mathbf{F}$

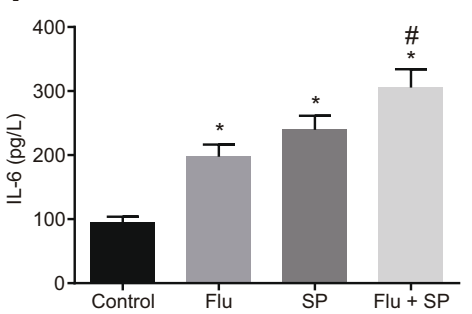

G

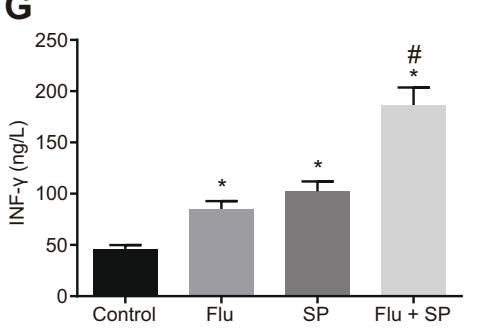

Fig. 3. Activation of the PI3K/AKT signaling pathway and aggravation of cellular inflammation during reinfection of Streptococcus pneumoniae in a mouse model after influenza virus infection. a, b Determination of Beclin1 and the PI3K/AKT signaling pathway-related proteins in mice by western blot analysis. $\mathbf{c}$, $\mathbf{d}$ The expression of Beclin1 in lung tissue of each group examined by immunohistochemistry $(\times 400)$. $\mathbf{e}-\mathbf{g}$ ELISA used to detect the expression of TNF- $\alpha$, IL-6, and IFN- $\gamma$ in bronchoalveolar lavage fluid of mice. $* p<0.05 v s$. the control group. The measurement data were expressed in the form of mean \pm standard deviation, and the comparison among multiple groups should be based on one-way analysis of variance, followed by Dunnett's post hoc test. The experiment was repeated 3 times. $N=10$. SP, Streptococcus pneumoniae; ELISA, enzyme-linked immunosorbent assay.

\section{Inhibition of the PI3K/AKT Signaling Pathway or Overexpression of Beclin1 Inhibits Reinfection of S. pneumoniae After Influenza Virus Infection}

PI3K inhibitor or Beclin1 lentivirus vector was used in nasal cavity inhalation to evaluate the expression of related protein and lung tissue injury in mice. The results of RT-qPCR showed that the expression of Beclin1 in lung tissue of mice in the oe-Beclin1 group was significantly higher than that in the oe-NC group $(p<0.05$; Fig. $4 a)$, suggesting that lentivirus infection was effective. Based on the results of western blot analysis, it showed that the levels of $\mathrm{p}-\mathrm{AKT} / \mathrm{AKT}$ and $\mathrm{p}-\mathrm{mTOR} / \mathrm{mTOR}$ in the PI3K inhibitor $+\mathrm{SP}$ and PI3K inhibitor + Flu + SP group were significantly lower than those in the DMSO + SP or DMSO + Flu $+\mathrm{SP}$ group, while the expression of Beclin1 significantly increased (all $p<0.05$; Fig. 4b, c). Besides, the S. pneumoniae bacteria in lung tissue of mice in each group at $48 \mathrm{~h}$ after infection was detected, and the corresponding results suggested that compared with the DMSO + Flu + SP group, the bacterial count in the PI3K inhibitor + Flu + SP group was significantly lower than that in the DMSO + $\mathrm{Flu}+\mathrm{SP}$ group $(p<0.05)$. Relative to the oe-NC $+\mathrm{Flu}+$ $\mathrm{SP}$ group, the number of bacteria in lung tissue of mice in the oe-Beclin $1+$ Flu + SP group decreased significantly $(p<0.05$; Fig. 4 d). Meanwhile, the activity of MPO was measured at $48 \mathrm{~h}$ after infection. In comparison to the $\mathrm{DMSO}+\mathrm{SP}$ group or DMSO + Flu + SP group, the activity of MPO in the PI3K inhibitor + SP group or the $\mathrm{PI} 3 \mathrm{~K}$ inhibitor $+\mathrm{Flu}+\mathrm{SP}$ group significantly decreased $(p<0.05)$. Furthermore, in contrast to the oe-NC + SP group or the oe-NC + Flu $+\mathrm{SP}$ group, the activity of MPO in lung tissue of mice in the oe-Beclin $1+$ SP group or the oe-Beclin $1+$ Flu + SP group decreased significantly $(p<0.05$; Fig. $4 \mathrm{e})$. The pathological changes of lung tissue of mice were determined by HE staining, which showed that compared to the DMSO + SP group or the DMSO + $\mathrm{Flu}+\mathrm{SP}$ group, as well as the oe-NC + SP group or the oe$\mathrm{NC}+\mathrm{Flu}+\mathrm{SP}$ group, the degree of injury in the lung tissue of mice significantly decreased in the PI3K inhibitor + SP group or the PI3K inhibitor + Flu + SP group, as well as in the oe-Beclin1 + SP group or the oe-Beclin $1+$ Flu + SP group (Fig. 4f). Considering inflammatory response, we found that the expression of TNF- $\alpha$, IL- 6 , and IFN- $\gamma$ decreased in lung tissues of mice in the PI3K inhibitor + $\mathrm{SP}$ group or the PI3K inhibitor $+\mathrm{Flu}+\mathrm{SP}$ group relative to the DMSO + SP group or DMSO + Flu + SP group (all $p<0.05$ ). The expression of TNF- $\alpha$, IL- 6 , and IFN- $\gamma$ also decreased in lung tissues of mice in the oe-Beclin $1+$ SP 
A
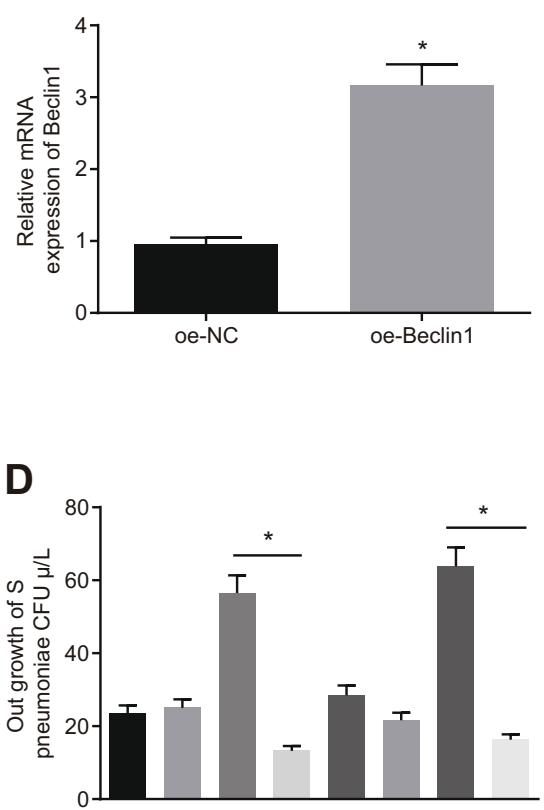

$\mathbf{F}$

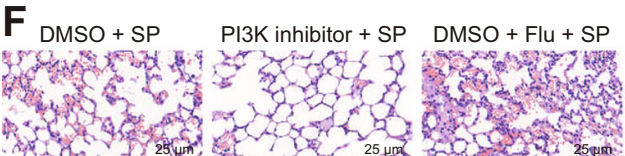

की

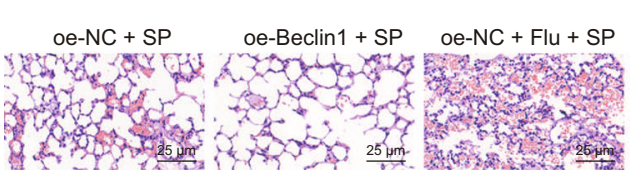

H

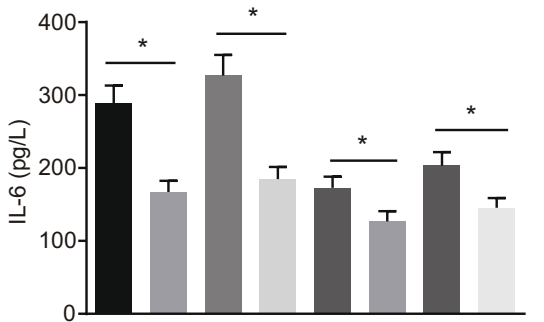

B

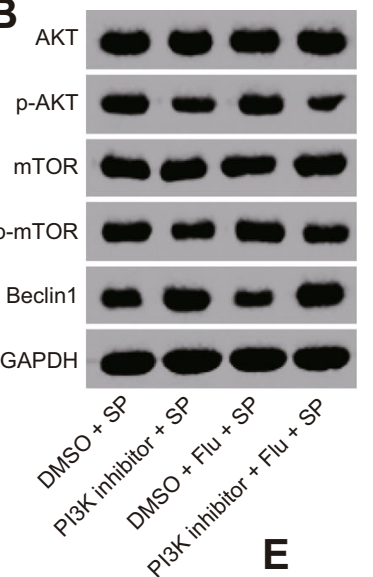

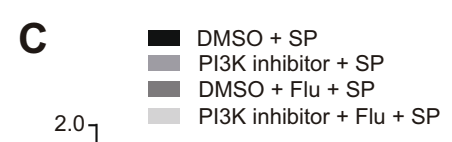
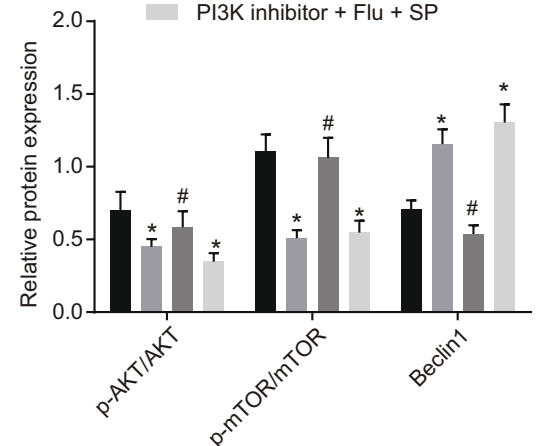

DMSO + SP

PI3K inhibitor + SP

$\mathrm{DMSO}+\mathrm{Flu}+\mathrm{SP}$

PI3K inhibitor + Flu + SP

oe-NC + SP

oe-Beclin1 + SP

oe-NC + Flu + SP

oe-Beclin1 + Flu + SP

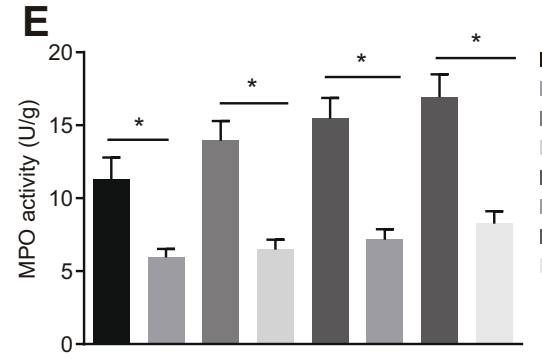

$\mathrm{DMSO}+\mathrm{SP}$

PI3K inhibitor + SP

DMSO + Flu + SP

$\mathrm{PI} 3 \mathrm{~K}$ inhibitor + Flu + SP

oe-NC + SP

oe-Beclin1 + SP

oe-NC + Flu + SP

oe-Beclin1 + Flu + SP

PI3K inhibito

$\mathrm{Flu} \stackrel{+}{+} \mathrm{SP}$

G

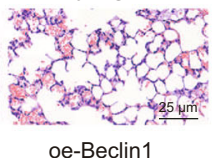

oe-Beclin1

$\mathrm{Flu}+\mathrm{SP}$
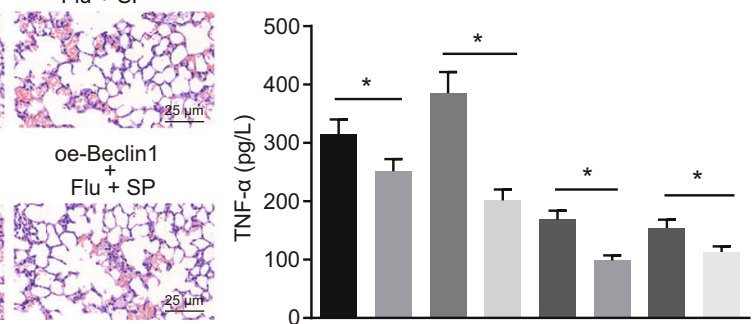

DMSO + SP

PI3K inhibitor + SP

DMSO + Flu + SP

PI3K inhibitor + Flu + SF

oe-NC + SP

oe-Beclin1 + SP

oe-NC + Flu + SP

oe-Beclin1 + Flu + SP

I

DMSO + SP

PI3K inhibitor $+\mathrm{SP}$

$\mathrm{DMSO}+\mathrm{Flu}+\mathrm{SP}$

PI3K inhibitor + Flu + SP

oe-NC + SP

oe-Beclin1 + SP

oe-NC + Flu + SP

oe-Beclin1 + Flu + SP

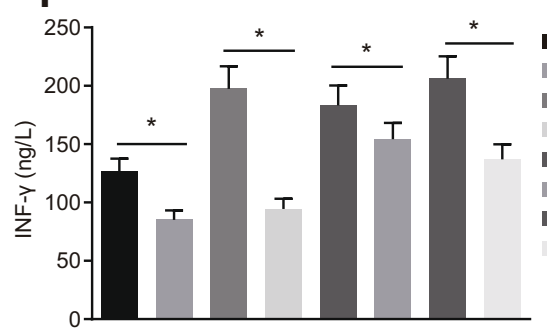

DMSO + SP

PI3K inhibitor $+\mathrm{SP}$

DMSO + Flu + SP

PI3K inhibitor + Flu + SP

oe-NC + SP

oe-Beclin1 + SP

oe-NC + Flu + SP

oe-Beclin1 + Flu + SP

Fig. 4. Inhibition of the PI3K/AKT signaling pathway or overexpression of Beclin1 suppress reinfection of Streptococcus pneumoniae after influenza virus infection. a Determination of mRNA expression of Beclin1 by RT-qPCR. b, $\mathbf{c}$ Determination of the PI3K/AKT signaling pathway-related proteins in mice by western blot analysis, ${ }^{*} p<0.05 v s$. the DMSO + SP group, ${ }^{*} p<0.05 v s$. the DMSO + Flu + SP group. d Measurement of Streptococcus pneumoniae in bronchoalveolar lavage fluid of mice. e Measurement of MPO activity in bronchoalveolar lavage fluid of mice. f Observation of pathological changes of lung tissue in mice by HE staining $(\times 400)$. g-i ELISA used to determine the expression of TNF- $\alpha$, IL- 6 , and IFN- $\gamma$ in bronchoalveolar lavage fluid of mice. ${ }^{*} p<0.05$ vs. the NC group or the DMSO group in $\mathbf{d}-\mathbf{i}$. The measurement data were expressed in the form of mean \pm standard deviation, and the comparison between two groups was analyzed by the nonpaired $t$ test. The experiment was repeated 3 times. $N=10$. SP, Streptococcus pneumoniae; ELISA, enzymelinked immunosorbent assay; RT-qPCR, reverse transcription quantitative polymerase chain reaction; NC, negative control; MPO, myeloperoxidase; HE, hematoxylin and eosin; DMSO, dimethyl sulfoxide. 


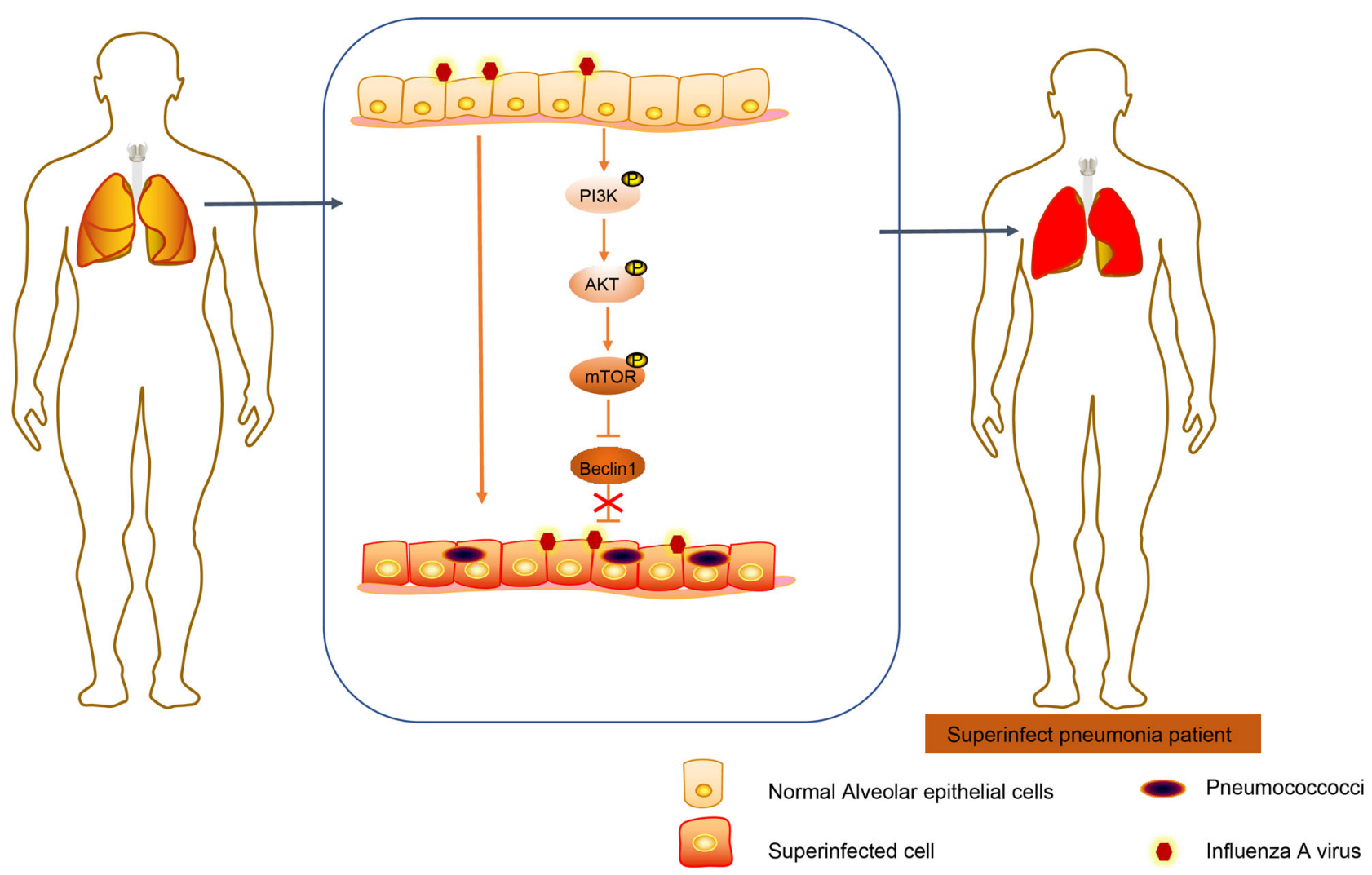

Fig. 5. The potential molecular mechanism of Beclin1-PI3K/AKT axis in reinfection of S. pneumoniae after influenza A virus. In pneumonia, influenza A virus infection can promote the reinfection of S. pneumoniae by promoting the activation of PI3K/AKT signaling pathway and downregulation of Beclin1 in bronchoalveolar lavage fluid.

group or the oe-Beclin $1+\mathrm{Flu}+\mathrm{SP}$ group in comparison to the oe-NC $+\mathrm{SP}$ group or the oe-NC $+\mathrm{Flu}+\mathrm{SP}$ group (all $p<0.05$; Fig. $4 \mathrm{~g}-\mathrm{i}$ ). These results suggest that the inhibition of the PI3K/AKT signaling pathway or overexpression of Beclin1 in a mouse model can inhibit reinfection of $S$. pneumoniae after influenza virus infection and thus alleviate the degree of injury.

\section{DISCUSSION}

The gram-positive pathogen $S$. pneumoniae is regarded as one of the main causative agents of CAP, and the incidence of pneumococcal pneumonia has been decreased due to the development and widespread application of antibiotics and pneumococcal vaccine [30]. Due to the increasing resistance among respiratory pathogens, together with the fact that $S$. pneumoniae is considered as the most common etiologic agent that is identified in CAP, the strategies for antimicrobial therapy should no doubt based upon the causative pathogen, local resistance patterns as well as the risk factors for infection with resistant bacteria [3]. However, the causative agents of CAP have not been routinely determined in China, especially in some severe cases. In this present study, we aim to discuss the molecular mechanism of reinfection of bacteria plus virus, figuring out the role of Beclin1-PI3K/AKT axis in reinfection of S. pneumoniae after influenza A virus. Collectively, we came to a conclusion that the inhibition of the $\mathrm{PI} 3 \mathrm{~K} / \mathrm{AKT}$ signaling pathway or overexpressed Beclin1 is in favor of suppressing the reinfection of $S$. pneumoniae after influenza A virus infection in SCAP.

First of all, this study demonstrated that BALF sampling was superior to traditional sputum sampling in the detection of bacterial pathogens in pneumonia. BAL uses sterile fluid to wash pathogens directly. BAL has been widely applicable for adults and children in the determination of the reason of ventilator-associated pneumonia, nosocomial pneumonia, severe CAP, or recalcitrant pneumonia [14]. Additionally, BALF is suggested to be easily suitable for multiple detection methods, such as microscopy, aerobic culture, PCR as well as viral culture [15]. BAL 
is a noninvasive method, hence safe for patients. A previous study has suggested that BAL could be a useful tool in the identification of pathogens in ventilator-associated pneumonia, or in acute hypoxemic respiratory failure which requires noninvasive ventilation [31]. Another literature also has elucidated the utility of BAL acting as a less invasive option for the assessment of lung pathology, which could also aids in the diagnosis of Cytomegalovirus pneumonitis using molecular methods [32]. Therefore, BAL is an accurate and safe method to validate pathogens in pneumonia.

In addition, this present study also demonstrated that the expression of Beclin1 was downregulated and the PI3K/AKT pathway was activated in reinfection of pneumonia. Besides pneumonia, similar to our study, a study revealed that Beclin1 expression was markedly downregulated in cartilage tissue of osteoarthritis [33]. And it has been reported that Beclin1 is component in the PI3K complex and it is also a target of the kinase AKT [19]. Several studies have elucidated that the activation of PI3K happens in host cells during the invasive process by Streptococcus agalactiae (S. agalactiae), and the involvement of PI3K has been detected during the invasion process of $S$. agalactiae together with other human pathogens [34, 35], which suggests a responsive inflammation occur. A recent study has suggested that the activation of AKT is of great importance for engulfment of $P$. aeruginosa, a extracellular pathogen, and the loss of bacterial motility results in a corresponding loss of stimulation and phagocytosis of AKT [36]. Taken together, the PI3K/ AKT signaling pathway collaborates with Beclin1 to regulate pneumonia progression.

Furthermore, our study also indicated that the inhibition of the PI3K/AKT pathway or overexpression of Beclin1 inhibited the pneumonia symptoms after pathogen infection. Beclin1 regulates autophagy initiation through interaction with Atg14/ATG14L/Barkor (Complex I) and regulates vacuolar protein sorting, cytokinesis as well as receptor degradation by regulating Vps38/UVRAG (Complex II) [17]. And it is demonstrated that ectopic Beclin1 expression suppressed cancer cell proliferation in vitro and tumorigenic potential in vivo, thereby implying a role for autophagy in tumor suppression [18]. As for the $\mathrm{PI} 3 \mathrm{~K} / \mathrm{AKT} / \mathrm{mTOR}$ pathway, it is a signal transduction pathway that is implicated in the regulation of a great deal of cellular functions, such as cell proliferation, differentiation, adhesion, and invasion [37]. It is reported that the PI3K/AKT signaling pathway is frequently initiated through the regulation of trans-membrane receptor signaling, which is followed by activation of Src kinases to recruit and activate PI3K [38]. Kierbal et al. have observed that blockade of cellular PI3K activity is able to prevent $P$. aeruginosa entry into both kidney and HeLa epithelial cells [39]. Therefore, it is promising to target Beclin1-PI3K/AKT for pneumonia treatment.

\section{CONCLUSION}

In conclusion, the novel aforementioned findings asserted may open novel chapters for future SCAP treatments. Our study demonstrates that BAL is an accurate method for clinical diagnosis of pneumonia, and the PI3K/ AKT signaling pathway inhibition or Beclin1 is capable of suppressing the reinfection of $S$. pneumoniae after influenza A virus infection in SCAP (Fig. 5). However, the potential mechanism underlying the role of Beclin1 and the PI3K/AKT signaling pathway require further investigation in future following studies.

\section{ACKNOWLEDGMENTS}

This study was supported by the Qingdao Municipal Project. We acknowledge and appreciate our colleagues for their valuable efforts and comments on this paper.

\section{AUTHORS' CONTRIBUTIONS}

Zhaohui Yang, Xiaoguang Zou, and Peiqing Feng designed the study. Huaibing Zhan, Dani Xiong, and Jianmin Lang collated the data, carried out data analyses, and produced the initial draft of the manuscript. Zhaohui Yang contributed to drafting the manuscript. All authors participated in the revised manuscript, and have read and approved the final submitted manuscript.

\section{COMPLIANCE WITH ETHICAL STANDARDS}

Competing Interests. The authors declare that they have no competing interests.

\section{REFERENCES}

1. Torres, A., O. Sibila, M. Ferrer, E. Polverino, R. Menendez, J. Mensa, A. Gabarrús, J. Sellarés, M.I. Restrepo, A. Anzueto, M.S. Niederman, and C. Agustí. 2015. Effect of corticosteroids on treatment failure among hospitalized patients with severe communityacquired pneumonia and high inflammatory response: A randomized clinical trial. JAMA 313 (7): 677-686. 
2. Woodhead, M., C.A. Welch, D.A. Harrison, G. Bellingan, and J.G. Ayres. 2006. Community-acquired pneumonia on the intensive care unit: Secondary analysis of 17,869 cases in the ICNARC case mix programme database. Critical Care 10 (Suppl 2): S1.

3. File, T.M., Jr. 2004. Streptococcus pneumoniae and communityacquired pneumonia: A cause for concern. The American Journal of Medicine 117 (Suppl 3A): 39S-50S.

4. Lee, J.H., H.J. Kim, and Y.H. Kim. 2017. Is beta-lactam plus macrolide more effective than beta-lactam plus fluoroquinolone among patients with severe community-acquired pneumonia?: A systemic review and meta-analysis. Journal of Korean Medical Science 32 (1): 77-84.

5. Bi, J., J. Yang, Y. Wang, C. Yao, J. Mei, Y. Liu, J. Cao, and Y. Lu. 2016. Efficacy and safety of adjunctive corticosteroids therapy for severe community-acquired pneumonia in adults: An updated systematic review and meta-analysis. PLoS One 11 (11): e0165942.

6. Mandell, L.A., R.G. Wunderink, A. Anzueto, J.G. Bartlett, G.D. Campbell, N.C. Dean, S.F. Dowell, T.M. File Jr., D.M. Musher, M.S. Niederman, A. Torres, and C.G. Whitney. 2007. Infectious Diseases Society of America/American Thoracic Society consensus guidelines on the management of community-acquired pneumonia in adults. Clinical Infectious Diseases 44 (Suppl 2): S27-S72.

7. Ferrer, M., C. Travierso, C. Cilloniz, A. Gabarrus, O.T. Ranzani, E. Polverino, A. Liapikou, F. Blasi, and A. Torres. 2018. Severe community-acquired pneumonia: Characteristics and prognostic factors in ventilated and non-ventilated patients. PLoS One 13 (1): e0191721.

8. Bjarnason, A., G. Thorleifsdottir, A. Love, J.F. Gudnason, H. Asgeirsson, K.L. Hallgrimsson, et al. 2012. Severity of influenza A 2009 (H1N1) pneumonia is underestimated by routine prediction rules. Results from a prospective, population-based study. PLoS One 7 (10): e46816.

9. Jennings, L.C., T.P. Anderson, K.A. Beynon, A. Chua, R.T. Laing, A.M. Werno, et al. 2008. Incidence and characteristics of viral community-acquired pneumonia in adults. Thorax 63 (1): 42-48.

10. Tsolia, M.N., S. Psarras, A. Bossios, H. Audi, M. Paldanius, D. Gourgiotis, K. Kallergi, D.A. Kafetzis, A. Constantopoulos, and N.G. Papadopoulos. 2004. Etiology of community-acquired pneumonia in hospitalized school-age children: Evidence for high prevalence of viral infections. Clinical Infectious Diseases 39 (5): 681-686.

11. Lim, W.S., S.V. Baudouin, R.C. George, A.T. Hill, C. Jamieson, I. Le Jeune, et al. 2009. BTS guidelines for the management of community acquired pneumonia in adults: Update 2009. Thorax 64 (Suppl 3): iii1-ii55.

12. Lynch, I.J., and F.J. Martinez. 2002. Clinical relevance of macrolideresistant Streptococcus pneumoniae for community-acquired pneumonia. Clinical Infectious Diseases 34 (Suppl 1): S27-S46.

13. Cilloniz, C., S. Ewig, R. Menendez, M. Ferrer, E. Polverino, S. Reyes, et al. 2012. Bacterial co-infection with H1N1 infection in patients admitted with community acquired pneumonia. The Journal of Infection 65 (3): 223-230.

14. Tsai, C.M., K.S. Wong, W.J. Lee, K.S. Hsieh, P.L. Hung, C.K. Niu, and H.R. Yu. 2017. Diagnostic value of bronchoalveolar lavage in children with nonresponding community-acquired pneumonia. Pediatrics and Neonatology 58 (5): 430-436.

15. De Schutter, I., E. De Wachter, F. Crokaert, J. Verhaegen, O. Soetens, D. Pierard, et al. 2011. Microbiology of bronchoalveolar lavage fluid in children with acute nonresponding or recurrent community-acquired pneumonia: Identification of nontypeable Haemophilus influenzae as a major pathogen. Clinical Infectious Diseases 52 (12): 1437-1444.

16. Qin, Z., Y. Yang, H. Wang, J. Luo, X. Huang, J. You, et al. 2016. Role of autophagy and apoptosis in the postinfluenza bacterial pneumonia. BioMed Research International 2016: 3801026.
17. Rohatgi, R.A., J. Janusis, D. Leonard, K.D. Bellve, K.E. Fogarty, E.H. Baehrecke, et al. 2015. Beclin 1 regulates growth factor receptor signaling in breast cancer. Oncogene 34 (42): 5352-5362.

18. Jiang, L.C., S.Y. Huang, D.S. Zhang, S.H. Zhang, W.G. Li, P.H. Zheng, and Z.W. Chen. 2014. Expression of beclin 1 in primary salivary adenoid cystic carcinoma and its relation to Bcl-2 and p53 and prognosis. Brazilian Journal of Medical and Biological Research 47 (3): 252-258.

19. Wang, R.C., Y. Wei, Z. An, Z. Zou, G. Xiao, G. Bhagat, M. White, J. Reichelt, and B. Levine. 2012. Akt-mediated regulation of autophagy and tumorigenesis through Beclin 1 phosphorylation. Science 338 (6109): 956-959.

20. Oliveira, J.S.S., G.D.S. Santos, J.A. Moraes, A.M. Saliba, T.C. Barja-Fidalgo, A.L. Mattos-Guaraldi, et al. 2018. Reactive oxygen species generation mediated by NADPH oxidase and PI3K/Akt pathways contribute to invasion of Streptococcus agalactiae in human endothelial cells. Memórias do Instituto Oswaldo Cruz 113 (6): e140421.

21. Scrima, M., C. De Marco, F. Fabiani, R. Franco, G. Pirozzi, G. Rocco, et al. 2012. Signaling networks associated with AKT activation in non-small cell lung cancer (NSCLC): New insights on the role of phosphatydil-inositol-3 kinase. PLoS One 7 (2): e30427.

22. Salo, P., A. Ortqvist, and M. Leinonen. 1995. Diagnosis of bacteremic pneumococcal pneumonia by amplification of pneumolysin gene fragment in serum. The Journal of Infectious Diseases 171 (2): 479-482.

23. Michelow, I.C., K. Olsen, J. Lozano, N.K. Rollins, L.B. Duffy, T. Ziegler, J. Kauppila, M. Leinonen, and G.H. McCracken. 2004. Epidemiology and clinical characteristics of community-acquired pneumonia in hospitalized children. Pediatrics 113 (4): 701-707.

24. Livak, K.J., and T.D. Schmittgen. 2001. Analysis of relative gene expression data using real-time quantitative PCR and the 2(-Delta Delta C(T)) method. Methods 25 (4): 402-408.

25. Biacchesi, S., M.H. Skiadopoulos, L. Yang, B.R. Murphy, P.L. Collins, and U.J. Buchholz. 2005. Rapid human metapneumovirus microneutralization assay based on green fluorescent protein expression. Journal of Virological Methods 128 (1-2): 192-197.

26. Lauw, F.N., J. Branger, S. Florquin, P. Speelman, S.J. van Deventer, S. Akira, et al. 2002. IL-18 improves the early antimicrobial host response to pneumococcal pneumonia. Journal of Immunology 168 (1): $372-378$.

27. Rijneveld, A.W., S. Florquin, J. Branger, P. Speelman, S.J. Van Deventer, and T. van der Poll. 2001. TNF-alpha compensates for the impaired host defense of IL-1 type I receptor-deficient mice during pneumococcal pneumonia. Journal of Immunology 167 (9): $5240-5246$.

28. van der Sluijs, K.F., L.J. van Elden, M. Nijhuis, R. Schuurman, J.M. Pater, S. Florquin, et al. 2004. IL-10 is an important mediator of the enhanced susceptibility to pneumococcal pneumonia after influenza infection. Journal of Immunology 172 (12): 7603-7609.

29. Knapp, S., J.C. Leemans, S. Florquin, J. Branger, N.A. Maris, J. Pater, N. van Rooijen, and T. van der Poll. 2003. Alveolar macrophages have a protective antiinflammatory role during murine pneumococcal pneumonia. American Journal of Respiratory and Critical Care Medicine 167 (2): 171-179.

30. Hu, H., L. He, S. Yu, K. Yao, A. Dmitriev, J. Deng, C. Zhao, X. Shen, and Y. Yang. 2009. Streptococcus pneumoniae as a frequent cause of severe community-acquired pneumonia among children in Beijing. European Journal of Clinical Microbiology \& Infectious Diseases 28 (9): 1129-1132.

31. Lacroix, G., B. Prunet, J. Bordes, N. Cabon-Asencio, Y. Asencio, T. Gaillard, S. Pons, E. D'aranda, D. Kerebel, E. Meaudre, and P. Goutorbe. 2013. Evaluation of early mini-bronchoalveolar lavage 
in the diagnosis of health care-associated pneumonia: A prospective study. Critical Care 17 (1): R24.

32. Mane, A., P. Gujar, S. Gaikwad, T. Dhamgaye, and A. Risbud. 2017. Detection of cytomegalovirus in bronchoalveolar lavage fluid from HIV-positive individuals with community acquired pneumonia. Journal of Clinical and Diagnostic Research 11 (7): DC41-DC43.

33. Song, B., H. Song, W. Wang, H. Wang, H. Peng, J. Cui, R. Wang, H. Huang, W. Wang, and L. Wang. 2017. Beclin 1 overexpression inhibits chondrocyte apoptosis and downregulates extracellular matrix metabolism in osteoarthritis. Molecular Medicine Reports 16 (4): 3958-3964.

34. Burnham, C. A., S. E. Shokoples and G. J. Tyrrell 2007. Invasion of HeLa cells by group B streptococcus requires the phosphoinositide3-kinase signalling pathway and modulates phosphorylation of hostcell Akt and glycogen synthase kinase-3. Microbiology 153(Pt 12): 4240-4252.

35. Dowd, G.C., M. Bhalla, B. Kean, R. Thomas, and K. Ireton. 2016. Role of host type IA phosphoinositide 3-kinase pathway components in invasin-mediated internalization of Yersinia enterocolitica. Infection and Immunity 84 (6): 1826-1841.
36. Lovewell, R.R., S.M. Hayes, G.A. O'Toole, and B. Berwin. 2014. Pseudomonas aeruginosa flagellar motility activates the phagocyte PI3K/Akt pathway to induce phagocytic engulfment. American Journal of Physiology. Lung Cellular and Molecular Physiology 306 (7): L698-L707.

37. Fumarola, C., M.A. Bonelli, P.G. Petronini, and R.R. Alfieri. 2014. Targeting PI3K/AKT/mTOR pathway in non small cell lung cancer. Biochemical Pharmacology 90 (3): 197-207.

38. Hannigan, M.O., C.K. Huang, and D.Q. Wu. 2004. Roles of PI3K in neutrophil function. Current Topics in Microbiology and Immunology 282: $165-175$.

39. Kierbel, A., A. Gassama-Diagne, K. Mostov, and J.N. Engel. 2005. The phosphoinositol-3-kinase-protein kinase B/Akt pathway is critical for Pseudomonas aeruginosa strain PAK internalization. Molecular Biology of the Cell 16 (5): 2577-2585.

Publisher's Note Springer Nature remains neutral with regard to jurisdictional claims in published maps and institutional affiliations. 